\title{
FACE DETECTION USING CASCADE CLASSIFIER WITH 7 LAYERS BASED ON THE HUMANS' IMAGES
}

\author{
Jyoti Singh Jadon ${ }^{1 *}$, Mr. Dheerendra singh tomar ${ }^{2}$ \\ ${ }^{*}$ M. Tech (CSE), Maharana Pratap College of Technology, Gwalior (Madhya Pradesh), \\ ${ }^{2}$ Dept. of CSE, Pratap College of Technology, Gwalior (Madhya Pradesh), India \\ *1jyotisingh0188@gmail.com, ${ }^{2}$ id-dst0513@gmail.com
}

*Corresponding Author: -

Email ID - jyotisingh0188@gmail.com

\begin{abstract}
: -
Age of human can be inferred by distinct patterns emerging from the facial appearance. Humans can easily distinguish which person is elder and which is older between two persons. When inferring a person's age, the comparison is done with his/her face and with many people whose ages are known, resulting in a series of comparative series, and then judgment is done based on the comparisons. The computer based age classification has become particularly prevalent topics recently. In this paper age classification is done by using Support Vector Machine technique. In variety of applications SVM has achieved excellent generalization performance.
\end{abstract}

Keywords: - Age Classification, Aging, Face Support Vector Machine, SVM

\section{(c) $(\$)$}




\section{INTRODUCTION}

The human face's proportions and expressions are very important to identify origin, emotional tendencies, health qualities, and some social information. From the birth, our faces are important in the individual's social influence. A person's face is the repository of a lot of information such as age, gender and identity. Faces allow humans to estimate/classify the age of other persons just by looking at their face [1].

Human brain and mind has an extraordinary ability to identify different faces from

knowledge of appearance. Human brain is not so precise when it comes to the classification of age. Researchers who perform work in studying the process of age classification by humans comes to a decision that humans are not so precise in age classification hence the possibility of developing facial age classification/estimation methods presents an arousing interest and exciting direction. In the present experiment, we have tried to develop an algorithm and computer program which can classify human age according to features extracted from human facial images using Support Vector Machine (SVM).[4]

Facial features are used in many researches such as face recognition, gender classification, and facial expressions and so on but only few of them have been done on age classification.

\section{RELATED WORK}

Many efforts towards age classification have been tried and most of them give results for wide ranges of ages or classify the ages in groups such as child, adult and old.

Finding an appropriate method for age classification for getting more specific categories of age ranges is still a challenging problem. Thus we focus our research on predicting a more accurate age group.

To achieve our goal, we have to build a good database that will be used to train and test our proposed method. Then the next objective is to construct a proper SVM to model our problem. Since the focus of the current research paper is on age classification and not on other allied fields like face recognition, so we have narrowed down our image database to only the images of frontal faces.

We have taken care to include only such images in our dataset which are as clear as possible without and images without any external additions viz. glasses, beards, scar marks, cosmetics etc. Also the faces should conform to general average faces of the populations. We have avoided including such faces which deviates from general average face to a large degree or faces which have any kind of distortions. Another of our face selection criterion is diversity. We have tried to include different types of faces in our dataset so as to widen the scope our method and enhance the accuracy in age classification. Once our dataset is ready we will carry out training of our proposed method. After reasonable amount of training is completed we will proceed to the testing of the application by providing to it a set of new faces of known age, which are not part of the training dataset, and then to see with what accuracy the application is able to predict the age group.

Age classification can be viewed as a "special" pattern recognition problem. The process of age classification could figure in a variety of applications [1], viz. -access control, human machine interaction, ageinvariant identity verification, data mining and organization Age classification systems can be used for age based retrieval and classification of face images enabling in that way automatic sorting and image retrieval from e-photo albums and the internet.

Age classification shares numerous problems encountered in other typical face image interpretation tasks [1] such as:

- face detection,

- face recognition,

- facial expression, and

- gender recognition

Facial appearance distortions caused by different expressions, inter-person variation, lighting variation, face orientation and the presence of occlusions have a negative impact on the performance on automatic age estimation. However, when compared to other face image interpretation tasks, the problem of age classification/estimation displays additional unique challenges that include:

In certain cases differences in appearance between adjacent age groups are negligible, causing difficulties in the process of age classification. This problem is escalated when dealing with mature subjects.

Both the rate of aging and type of age-related effects varies from person to person and from environment to environment. For example, the amount of facial wrinkles may be significantly different for different individuals belonging to the same age group. As a result of the diversity of aging variation, the use of the same age classification strategy for all subjects may not produce adequate performance. Several factors could influence the aging process including race, gender and genetic traits. For this reason different age classification approaches may be required for different groups of subject.

\section{Pattern Recognition}

Pattern recognition can be defined narrowly as dealing with feature extraction and classification [2]. In a broad sense pattern recognition has been around since our initial antecedents learned which animals they could approach to hunt and which they should flee from. Although they probably never stopped to analyze it, they were doing classification based on features, size, length of teeth, temperament, etc.

A definition of pattern recognition then is field whose objective is to assign an object or event to one of the number of groups, based on features derived to emphasize commonalities. The term pattern recognition can be misguiding. The patterns connected with pattern recognition are not single instances of patterns in a signal - not an area of stripes in an image or an interval of sinusoids in a sound clip. Instead, they are patterns of features that repeat across different samples. 
For instance image of plowed field may have a stripe pattern whose feature can be found by Fourier analysis. Pattern recognition concerns not to the single stripe pattern, by which they are classified together.

Now let's focus on the word recognition. in a broad sense, recognition implies the act of associating a classification with a label. Using the Figure 1 below, that would say that those samples falling into upper right region are recognized as dogs and those in the lower left are recognized as cats [2]. Many pattern recognition systems can be divided into components such as the ones describe here. A sensor converts images or other physical inputs into signal information. The segment or separates sensed objects from background orfrom other objects.

A feature extractor measures object properties that are bring some advantage for classification. The traditional aim of the feature extractor is to characterize an object to be recognized by measurements whose value is very similar for objects in the same group, and very different for objects in different groups. This precedes to the idea of searching distinguishing features that are invariant to irrelevant changes of the input data. In general, features that describe properties such as shape, color and may kinds of texture are invariant to translation, rotation and scale.

The classifier uses these features to assign the sensed object to a group. The work of the classifier stage of a full system is to use the feature vector provided by feature extractor to assign the object to the group. The degree of problem of the classification depends on the variability in the characteristic values for the objects in the same group relative to the difference between characteristic values for objects in different groups.

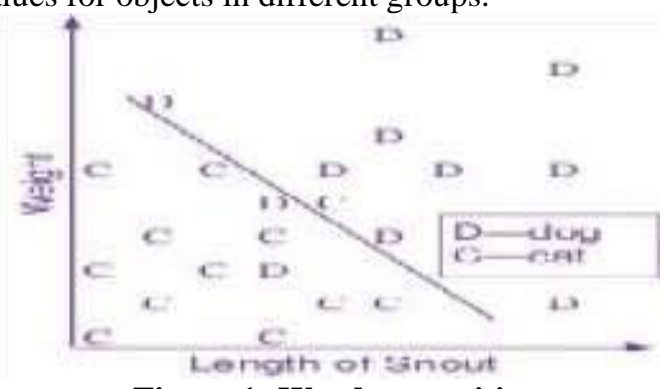

Figure 1: Word recognition

The variance of characteristic values for the objects in the same group may be due to complexity and noise present in the objects. We define noise in very general terms: any properties of sensed pattern which is not due to the true underlying model but instead to randomness in the world or the sensors. All the non-trivial decision and pattern recognition problems involve noise in some form. One problem that arises in practice is that it may not always be possible to determine the values of all of the features for a particular input.

\section{Image}

A digital image is a snapshot of an object and is reloaded in various ways as two-dimensional matrix or grid of pixels, where pixels are elements of a digital image having some intensity value corresponding to the reflected light from the object. There are two types of images i.e. gray scale and colored images where gray scale images are $2 \mathrm{D}$ matrix of pixels each pixel having some intensity value and colored image are also $2 \mathrm{D}$ matrix of pixels where every pixel have three intensity values each for a primary color i.e. red, green, blue (R,G,B).

A digital image is composed of pixels which can be thought of as small dots on the screen. A digital image is an instruction of how to color each pixel. A typical size of an image is 512-by512 pixels. In the general case we say that an image is of size $\mathrm{m}-\mathrm{by}-\mathrm{n}$ if it is composed of $\mathrm{m}$ pixels in the vertical direction and $\mathrm{n}$ pixels in the horizontal direction [3].

\section{Gray scale}

A gray scale (or gray level) image is simply an image in which the only colors are shades of gray. The reason for differentiating such images from any other sort of color image is that less information needs to be provided for each pixel. In fact a 'gray' color is one in which the red, green and blue components all have equal intensity in RGB space, and so specifying only a single intensity value for each pixel is necessary, as opposed to the three intensities needed to specify each pixel in a full color images.

Often, the gray scale intensity is stored as an 8bit integer giving 256 possible different shades of gray from black to white. If the levels are evenly spaced then the difference between successive gray levels is significantly better than the gray level resolving power of the human eye. Gray scale images are very common, in part because much of today's display and image capture hardware can only support 8-bit images. In addition, gray scale images are entirely sufficient for many tasks and so there is no need to use more complicated and harder-to-process color images.

A gray scale shows the number of intensity values that can be used to represent an image. An $n$ bit image have $2^{\text {nd }}$ intensity values in its gray scale whereas 1 bit image have only 2 intensity values in its gray scale. Most of the images are of 8 bit, so there are 256 intensity values in its gray scale where $o$ intensity value represent black, 256 intensity value represent white color and all values between 0 and 256 are intermediate gray intensities (see figure 2.1). [Gonzalez R.C and Woods R.E (2008)][4][5] 


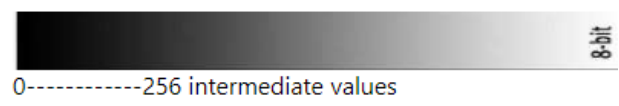

Figure 2: Grey scale of an 8 bit gray image.

\section{HistogramEqualization of Gray Scale Images}

In our current research we have used histogram equalization to enhance the image. Histogram equalization spreads the intensity values of the images over a larger range. It is used because it decreases the effect of different imaging conditions, for example different camera gains and it may also increase image contrast [6]. When analyzing faces it is important that there is as little variation due to external conditions (such as imaging conditions) as possible, so that the variations between the faces become more visible. This is the issue we are interested in. Histogram equalization is simple to implement and computationally inexpensive. The function that maps image pixel intensity to a histogram equalized value is

$$
s_{k}-\sum_{j=0}^{k} k
$$

$\mathrm{k}=0,1,2 \ldots \mathrm{L}-1$

Where $-\mathrm{S}_{\mathrm{k}}$ is histogram equalized intensity value for kth intensity value in the range $\mathrm{L}$ of total number of possible intensity values in theoriginal and target image.

$\mathrm{n}$ is the number of pixels in the original and target image, and

$\mathrm{nj}$ is the number of image pixels that have intensity value $\mathrm{i}$ in the original image.

Examples of histogram equalized face images with their original counter parts are shown in Figure 3 . As can be seen, face intensities look more uniform and the contrast has improved dramatically.

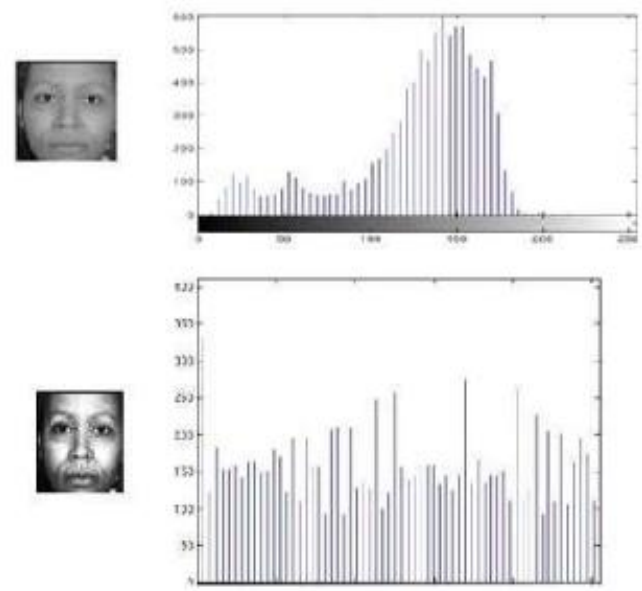

Figure 3: Original images are shown on the top and the corresponding histogram equalized face imagesare shown at the bottom of the figure. The histogram of each face image is shown at the right side of the image.

Although histogram equalization usually produces good results, it is worth noting that in some rare cases it does not work well. Such examples are shown in Figure 3. The result in Figure 3 is poor because there are a large number of black pixels (intensity value 0 ) in the original image and intensity values are concentrated at the low end of the intensity range. As a result, the values of the histogram equalized image are not spread over the whole intensity range. Instead, the lowest value is around 75 on a range of 0-255 [9].However, this is an extreme case and it was necessary to modify the example image manually to demonstrate the possibility of unsuccessful histogram equalization. Modification was done by adding black background to the left side of the image and by flattening dark intensity levels of the image. Histogram specification (also known as histogram matching) would work better in this case but, unlike histogram equalization, it requires manual parameter setup. From this it follows that histogram equalization is more useful in automatic face analysis systems.

\section{Classification}

Classification goals to build an efficient and effective model for predictiing the class labels for new samples/observations. The model is built on the training set of samples/observations and their class labels.

\section{Statistical Classification}

In machine learning, statistical classification is the problem of identifying the sub-group to which new samples belong, where identity of sub-group is unknown. On the basis of a training set of data containing samples whose sub-group is known. Therefore, these classifications will point out a variable behavior which can be studied by statistics [7][8]. 
Binary and Multi-class Classification Age classification can be thought of as composed of two separate problems Binary classification

Multi-class classification

In the binary classification problem as name indicate only two classes are involved, whereas in multi-class classification involves assigning an object to one of several classes. Since many classification methods have been developed for the binary classification, multi-class classification often requires the combined use of multiple binary classifiers.

\section{Overfitting}

The concept of over fitting is most important in machine learning algorithms. Over fitting means fitting the training data too much which may allow perfect classification of only the training data that may increase the performance of classification, but it is unlikely that it will perform well on new patterns. It degrades the generalization of performance.

\section{Review}

Age estimation techniques fall within two main approaches.

\section{Estimation on the basis of a set of facial features}

According to this approach, the problem is treated as a standard classification problem. And we solve using standard classifiers where age classification is performed by assigning a set of facial features to an age group.

Estimation on the basis of aging process

According to this approach, as an option, age estimation techniques that depend on the modeling of the aging process have been developed.

\section{MATERIALS AND METHODS}

Data collection can account for large part of the cost of development of a pattern recognition system. The development of accurate age classification systems requires the existence of appropriate data sets suitable for training and testing.

Dataset should contain multiple images showing the same subject at different agescovering a wide age range. Since aging is a type of facial variation that cannot be controlled directly by human, the collection of such data set requires the use of images captured in the past.

Currently there are three publicly available data sets IFDB, MORPH and FG-NET but none of them fulfill all the requirements for a data set suitable for age classification. The next generation of such datasets is called FSAR. This dataset tries to overcome exiting shortcoming and it will be released very soon. We collected the face image samples from websites as well as captured from mobile phones and digital cameras. Our dataset contained 432 images of different age groups, shown in the table [10]

\begin{tabular}{|c|c|c|c|c|}
\hline & $\begin{array}{l}\text { Children } \\
(1-15)\end{array}$ & $\begin{array}{l}\text { Adult } \\
(16-40)\end{array}$ & $\begin{array}{l}\text { Old } \\
(4180)\end{array}$ & Total \\
\hline Training Set & 120 & 120 & 120 & 360 \\
\hline $\begin{array}{l}\text { Testing Set } \\
\text { Database }\end{array}$ & 24 & 24 & 24 & 72 \\
\hline
\end{tabular}

Among many available face databases around the world, two of them include significant sets for aging individuals but not fulfill all the requirements used for this experiment and I have collected some of images from the http://www.facerec.org/databases/.

\section{Method}

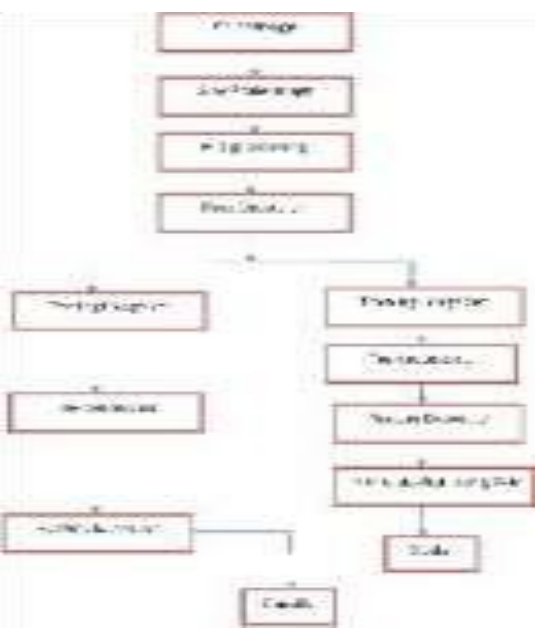

In age classification we use the following steps to perform the classification - convert the color and gray scale images to gray scale images, preprocessing, face detection, extract the features to train the classifiers and at last we perform the classification on test faces. The overview of the proposed method in age classification is summarized in the following figure: 
General Approach for Age Classification using SVM, First we perform the pre-processing operations on the input gray scale image. These operations are histogram equalization, intensity normalization. Histogram equalization and intensity normalization are operated on the image due to varying lighting conditions it enhance the image contrast and then frontal face of an image is extracted using its Haar cascade face detector also known as Viola-Jones Method[8] with the help of OpenCV library. And then again pre-process the frontal face of image using histogram equalization and intensity normalization. A training dataset is created by extracting the features from the faces. Then a classifier is trained with feature and the labeled data set pair and formed the model.

For a test image, the features are extracted in the same way. The model uses these features to classify the age group of the person. Convert Color image to Gray scale image

Gray value for pixel $i$ in an image is linear combination of three intensity values of three primary colors (Red, green, blue i.e. RGB) corresponding to pixel $i$.

Gray value $(\mathrm{i})=0.2989 * \mathrm{R}(\mathrm{i})+0.5870 * \mathrm{G}(\mathrm{i})+$

$0.1140 * \mathrm{~B}(\mathrm{i})$

[Source: MATLAB help] eq. (3.1)

Where

Gray value (i) = gray level value for pixel i.

$\mathrm{R}(\mathrm{i})=$ Intensity of red color in pixel i .

$\mathrm{G}(\mathrm{i})=$ Intensity of green color in pixel i . B(i) = Intensity of blue color in pixel i .

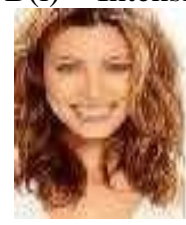

(a)

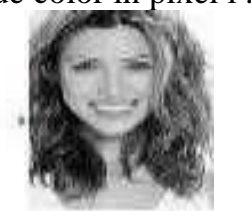

(b)

Figure 4 (a) : Color image Figure 4 (b) : Gray scale image.

\section{Face Detection}

As is described before face detection is done with the help of OpenCV library [17]. Face detection techniques are used to determine the location of human faces in a gray scale images while ignoring other objects in the image. There are wide variety of implementations and we use the OpenCV implementation that uses haar features. we used face detection algorithm for the facial feature extraction.

The description of the algorithm is as followViolaJones proposed the use of Haar-like features which can be computed efficiently with integral image. Figure 5 shows four types of Haar-like features that are used to encode the horizontal, vertical and diagonal intensity information of face images at different position and scale.

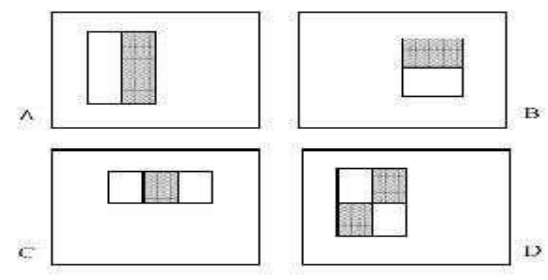

Figure 5: Haar-like features

The Haar-like features are computed as the difference of dark and light regions. They can be considered as features that collect local edge information at different orientation and scale. The set of Haar-like features is large, and only a small amount of them are learned from positive and negative examples for face detection.

Figure 6: Rectangular features. Example: For A, The value of a two-rectangle feature is the difference between the sums of the pixels within two rectangular regions.

Integral Image

Preprocess: normalize each image by dividing each pixel value by the standard deviation of the image. The value of the integral image at point $(x ; y)$ is the sum of all the pixels above and to the left.

$$
\begin{aligned}
& u(x, y)=\sum_{x^{\prime} \leq, x, y^{\prime} \leq y} i\left(x^{\prime}, y^{\prime}\right) \\
& \text { Where, } t(x, y) \text { is the integral image and } \\
& \text { the original image. } \\
& \text { Using the following pair of recurrences: } \\
& s[x, y)=s(x, y-1)+i(x, y) \\
& i[x, y)=i i(x-1, y)+s(x, y) \\
& i(x, y)
\end{aligned}
$$

The integral image can be computed in one pass over the original image. 


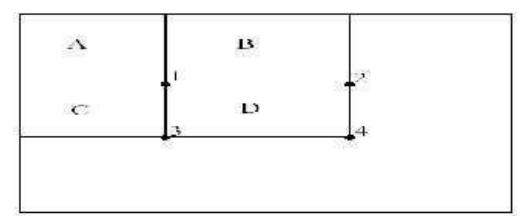

\section{Figure 6: Sum of the pixels within rectangle}

In the Figure 6 above, the sum of the pixels within rectangle D can be computed with four array references. The value of the integral image at location 1 is the sum of the pixels in rectangle $\mathrm{A}$. The value at location 2 is $\mathrm{A}+\mathrm{B}$, at location 3 is $\mathrm{A}$ $+C$, and at location 4 is $A+B+C+D$. The sum within D can be computed as $4+1-(2+3)$.

Using the integral image, the rectangular features can be calculated more efficiently.

\section{Adaboosting}

Boosting is a general method for improving the accuracy of any given learning algorithm. One can use it to combine simple "rules" (or weak learner), each performing only slightly better than random guess, to form an arbitrarily good hypothesis. Viola and Jones employed Adaboost (an adaptive boosting method) for object detection and got good performance when applying to human face detection [8].

\section{Classification using Support Vector Machine}

SVM (Support Vector Machine) are a useful technique for data classification. As in the proposed method (above figure) for SVM classification data is separated in training and testing sets. Each image in the training set contains one target value (i.e. the class labels) and several attributes (i.e. Features or observed variables). The aim if SVM is to train the classifier using training data, and to produce a model which predicts the class labels for the test data when the input is only test data.

For the training set is linearly non-separable then it is mapped to high-dimensional feature space. This projection into high dimensional feature space is efficiently performed by using kernels. The kernel $\mathrm{K}$ is defined as -

$\aleph\left(x_{i}, x_{j}\right)=\not\left(x_{i}\right)^{T} \mid \emptyset\left(x_{j}\right)$

For this project, a polynomial kernel is used which is defined as -

$\mathcal{E}\left(x_{i,}, x_{j}\right)=\left(\gamma x_{i}^{T} x_{j}-r\right)^{d}, y>0$

Here $\boldsymbol{V}, \boldsymbol{x}$ and $\boldsymbol{d}$ are kernel parameters which controls the performance of the classifier.

The following steps are taken for the classification in MatLab.

1.Given data are transformed in the format of SVM package (for example in the MatLab all data are taken as a separate matrix and corresponding group is taken as separate matrix).

A MatLab function libsvmread reads the file in LIBSVM format: [Class_labels, Data_Matrix] = libsvmread(,data.txt,). Output of the libsvmread is Label 1:feature1 2:feature2 3:feature3 etc.

1.Perform scaling on the data 2.Polynomial function taken as a kernel.

3.10-cross validation and find the best parameter $\mathrm{C}$ and $\gamma$.

4. Whole training set is trained by best parameter of $\mathrm{C}$ and $\gamma$. 4.Test Scaling

Before using SVM, scaling of data is very important. There are advantages when we scale the data. First, the main advantage of scaling is to avoid attributes in greater numeric ranges dominating those in smaller numeric ranges. Second, another advantage is to avoid numerical difficulties during the calculation. Kernel values depend on inner product of features vector, so polynomial kernel and linear kernel causes numerical problem if we do not perform scaling on data. The range of scaling each attribute is $[1,+1]$ or $[0,1]$.

\section{Cross Validation}

Cross validation is a statistical method of evaluating and comparing learning algorithms by dividing data into two partitions:

First is used to learn or train a model, and another is used to validate the model. The basic form of cross validation is $v$ fold cross validation. In $v$-fold cross validation the data is first partitioned into $v$ equal sized folds. Then $v$ iterations of training and validation is performed such that within each iteration a different fold of data is held-out for validation while remaining v-1 folds are used for learning. The advantage of this method over repeated random sub-sampling is that all observations are used for both training and validation, and each observation is used for validation exactly once. Using the cross-validation procedure, over-fitting problem can be prevented.

\section{Steps in Age Classification}

Following steps are implemented for age classification -

Step 1: Convert color or gray scale images into gray scale images.

Step 2: And then pre-process the images using

Histogram Equalization and intensity normalization techniques.

Step 3: Extract the frontal face of the image using 
Viola-Jones Method with the help of OpenCV library.

Step 4: Pre-processing and feature extraction.

Step 5: Create a Model using SVM classifier. Step 6: Predict the age group with the help Trained Model.

\section{RESULTS AND DISCUSSION}

The training set consists of 360 faces, 120 each of age group child, adult and old person faces. The test data have 72 faces, 24 each of age group child, adult and old person faces. The average accuracy of 10fold cross validation of SVM classifier using polynomial kernel is $71.7361 \%$.

When the training set is directly given to the libSVM [16] routine, depending on the training set and testing set, the accuracy of the SVM classifier is varied. The average accuracy for the age group of child, adult and old using combination of different training and testing set are approximately $83.3333 \%, 68.75 \%$ and $61.4583 \%$ respectively. The result obtained from the first trial is shown in table 1 and results obtained from the other trials are shown in appendix $\mathrm{H}$.

The experimental result of first trial is shown in the Table 2.

Table 2: Experimental Results

\begin{tabular}{|l|l|l|l|l|l|}
\hline $\begin{array}{l}\text { Input } \\
\text { Age } \\
\text { Group }\end{array}$ & $\begin{array}{l}\text { Ch } \\
\text { ild }\end{array}$ & Adult & & Old & $\begin{array}{l}\text { Age } \\
\text { Prediction } \\
\text { Rate }\end{array}$ \\
\hline Child & 18 & 3 & & 3 & $75 \%$ \\
\hline Adult & 4 & 16 & & 4 & $66.6667 \%$ \\
\hline Old & 2 & 5 & & 17 & $\begin{array}{l}70.8333 \\
\%\end{array}$ \\
\hline
\end{tabular}

The results obtained from the first trial are given below. Training set: Total $=360$

Child $=120$

Adult $=120$ Old $=120$ A- Test: total $=24 ; 18$ from class 0 .

Output Predicted Label:

0100000200200100002001

00

Accuracy: $18 / 24=75 \%$

B- Test: total $=24 ; 16$ from class 1 . Output Predicted Label:

200111112121021111111

110

Accuracy: $16 / 24=66.6667 \%$ C- Test: total $=24$; all 17 from class 2 Output class label:

2112222121222222202022

12

Accuracy: $17 / 24=70.8333 \%$

\section{CONCLUSION}

We proposed a method for age classification using facial features based on Support Vector Machine [SVM]. We classified the age into three age groups such as child, adult and old. The development process includes data collection, feature extraction and finally training and testing by the system Support Vector Machine. To train and test our system, we used the dataset taken from the websites.

There are 432 gray-scale facial images of each size 100*100 used for experiment. 360 images are used to train the SVM classifier. And the rest of images are used to evaluate the performance of the system.

For the test images, the correct rate for distinguishing child is $83.3333 \%$, adult $68.75 \%$ and old $61.4583 \%$. After systematic examination the factors that affect the performance of the classifier, some important are summarized as follows.

1. Facial wrinkles are usually removed by the photographer or due to some cosmetics i.e. men and women use cosmetic creams on their faces that cause problems to extract the exact features.

2. For some images light source are too strong so that some important features are lost. 3. Due to glasses and beards are also some important features are lost.

The performance of the classifier is dependent strongly on the nature of the training and testing data sets.

\section{REFERENCES}

[1]. 1.http://www.scholarpedia.org/article/Facial_Age_Es timationinternational association for pattern recognition Volume 25 Number 1 Winter 2003. http://amath.colorado.edu/computing/Matlab/T utorial /ImageProcess.html

[2]. Gonzalez R.C and Woods R.E (2008), Digital Image Processing Third edition, Pearson Education. Gonzalez R.C , Woods R.E and Eddins L. (2008), " Digital Image Processing Using MATLAB", Pearson Education. 
[3]. Erno Makinen. Introduction to Computer Vision from Automatic Face Analysis Viewpoint,

[4]. Department of Computer Sciences University of Tampere, Finland. Duda R. and Hart P. Pattern Classification and Scene Analysis, Wiley, New York 1973.

[5]. Nello Cristianini and John Shawe-Taylor, An Introduction to Support Vector Machines and Other Kernelbased Learning Methods, Cambridge University Press, 2000.

[6]. Tan PN, et al. Introduction to Data Mining (First Edn) 2005, Addison Wesley.

[7]. Y.H. Kwon and N. da Vitoria Lobo. Age Classification from Facial Images, Computer Vision and Image Understanding Journal 74(1), pp. 1-21, 1999.

[8]. Lanitis, C. J. Taylor and T.F. Cootes. Toward Automatic Simulation of Aging Effects on Face Images, IEEE Transactions of pattern analysis and machine intelligence, 24(4), pp 442-455, 2002.

[9]. Lanitis, C. Draganova C. and C. Christodoulou C. Comparing Different classifiers for automatic Age Estimation,. IEEE Transactions on Systems Man and Cybernetics, Part B,34(1), pp 621-629, 2004.

[10]. Geng, Z.H. Zhou and K. Smith-Miles. Automatic Age Estimation Based on Facial Aging Patterns, IEEE Transactions of Pattern Analysis and Machine Intelligence, 29(12), pp. 2234-2240, 2004.

[11]. 11. Y. Fu and T.S. Huang. Human Age Estimation with Regression on Discriminative Aging Manifold, IEEE Transactions on Multimedia, 10(4), pp. 578-584, 2008.

[12]. J.G. Wang, W. Y. Yau and H.L. Wang. Age Categorization via ECOC with Fused Gabor and LBP Features, Procs. Of the IEEE Workshop on Applications of Computer Vision(WACV), pp. 313318, 2009.

[13]. J. Suo, T. Wu, S. Zhu, S. Shan, X. Chen and W. Gao. Design Sparse Features for Age Estimation using Hierarchical Face Model, Proc. of the $8^{\text {th }}$ IEEE International Conference on Automatic Face and Gesture Recognition, 2008.

[14]. Viola, P. and Jones, M. Robust real-time face detection, International Journal of Computer Vision 57(2), pp. 137154, 2004.

[15]. Viola, P. and Jones, M. Rapid Object Detection using a Boosted Cascade of Simple Features, CVPR 2001.

[16]. http://en.wikipedia.org/wiki/MATLAB.

[17]. http://www.netlib.org/lapack/ 18. Chih-Chung Chang and Chih-Jen Lin. LIBSVM: a library for support vector machines, 2001. Softwareavailable at http://www.csie.ntu.edu.tw /cjlin/libsvm.http://en.wikipedia.org/wiki/ Statistical_classification. 